\title{
BIENESTAR ASOCIADO A LA JUBILACIÓN EN DOCENTES UNIVERSITARIOS DE LATINOAMÉRICA
}

\section{THE WELFARE ASSOCIATED WITH THE RETIREMENT OF THE UNIVERSITY TEACHING STAFF IN LATIN AMERICAN}

\author{
Mercedes Angélica García Pazmiño ${ }^{1}$ \\ Marco Gamboa Proaño² \\ Nelson Marcelo Quimbita ${ }^{3}$ \\ Luis Cornelio Castillo 4 \\ María José Centeno ${ }^{5}$
}

\author{
Recibido: 2019-08-03 / Revisado: 2019-10-15 / Aceptado: 2019-10-25 / Publicado: 2020-01-01
}

Forma sugerida de citar: García-Pazmiño, M. A., Gamboa-Proaño, M., Quimbita, N. M., Castillo, L. C. y Centeno, M. J. (2020). Bienestar asociado a la jubilación en docentes universitarios de Latinoamérica. Retos de la Ciencia. 4(8), pp. 25-37. https://doi.org/10.53877/rc.4.8.20200101.03

\section{RESUMEN}

La jubilación es un proceso social, una imposición administrativa en función de la edad y años laborados; esta población se convierte en un grupo vulnerable por el deterioro de las facultades físicas y mentales propias del ciclo evolutivo; por tanto, la jubilación tiene impacto en la salud pública. El presente estudio centró su atención en la comunidad de docentes universitarios jubilados de la universidad pública del Ecuador y planteó como objetivo analizar el bienestar psicológico asociado a la jubilación. Estudio descriptivo, cuantitativo, diseño transversal, correlacional, noexperimental. Se aplicó Escala de Bienestar Psicológico y el Cuestionario MOS de Apoyo Social. Los resultados caracterizaron el perfil sociodemográfico de 725 docentes jubilados en el período 2012-2017. Según su condición, tienen un promedio de edad 67 años; $45 \%$ viven con su pareja y el resto con otros familiares. Se determinó un grado elevado de bienestar psicológico, destacándose las dimensiones relaciones positivas con otros, dominio del entorno; a diferencia de propósito en la vida que reflejó un grado bajo. Asimismo, se demostró un elevado índice de apoyo social. La matriz de correlaciones debeló alta correlación entre las dimensiones de las variables.

Palabras Claves: bienestar psicológico, jubilación, docentes universitarios, apoyo social.

\footnotetext{
1 Doctora en Ciencias de la Salud en el Trabajo (PhD), Docente en la Universidad Central del Ecuador. Ecuador. E-mail: garcia.mercy@hotmail.com

2 Máster en Educación Infantil y Educación Especial, Docente en la Universidad Central del Ecuador. Ecuador, Email: magamboa@uce.edu.ec

${ }^{3}$ Máster en Ciencias Psicológicas con mención en Psicología Deportiva. Municipio del Distrito Metropolitano del Quito. Ecuador, E-mail: nelsonquimbita@yahoo.es

4 Maestro en Ciencias con especialidad en Probabilidad y Estadística. Docente en la Universidad Central del Ecuador. Ecuador, E-mail: Iccastillo@uce.edu.ec

${ }^{5}$ Psicóloga Clínica. Estudiante en la Universidad Central del Ecuador. Ecuador. E-mail: mjcenteno@uce.edu.ec
} 


\section{ABSTRAC}

Retirement is a social process, an administrative imposition based on age and years worked; this population becomes a vulnerable group due to the deterioration of the physical and mental faculties characteristic of the evolutionary cycle; therefore, retirement has an impact on public health. The present study focused its attention on the community of retired university professors from the public university of Ecuador and aimed to analyze the psychological well-being associated with retirement. Descriptive, quantitative, cross-sectional, correlational, non-experimental study. The Psychological Well-being Scale and the MOS Social Support Questionnaire were applied. The results characterized the socio-demographic profile of 725 retired teachers in the 2012-2017 period. According to their condition, they have an average age of 67 years; $45 \%$ live with their partner and the rest with other relatives. A high degree of psychological well-being was determined, highlighting the positive relationships with other dimensions, domain of the environment; unlike life purpose that reflected a low grade. Likewise, a high index of social support was demonstrated. The correlation matrix debuted a high correlation between the dimensions of the variables.

Keywords: psychological well-being, retirement, university teachers, social support.

\section{INTRODUCCIÓN}

La jubilación es una transición que implica cambios en los roles y relaciones sociales, rutinas diarias, ingreso económico y salud; puede promover en el jubilado un sentido de bienestar al retirarse de una actividad laboral demandante o estresante, lo que conlleva a pasar más tiempo con familiares y amigos; y realizar otras actividades de ocio satisfactorias.

Contrariamente a este criterio Mesa, Pachón y Puerto (2016) conciben a la jubilación como el retiro laboral que supone una pérdida de poder adquisitivo y un descenso en la autoestima, pues experimentan el fin de un ciclo vital y con él las percepciones de autoridad y productividad asociadas al trabajo, se extinguen; estos afectos negativos, dependen de las condiciones del individuo y su contexto.

Asimismo, Sánchez (2000), aborda a la jubilación como un derecho y un logro social que puede llegar a producirse en distintos momentos de la vida, bien sea de manera voluntaria o por imperativos varios. A su vez, el autor manifiesta que la jubilación es la "auténtica clave de bóveda de nuestra sociedad"; a lo que se suma una falta de sensibilidad por parte de la sociedad para atender a este grupo poblacional vulnerable, notándose de manera general una pérdida del respeto y consideración.

La jubilación está ligada a los procesos de envejecimiento etapa de cambios físicos, cognitivos y emocionales; cuadro que puede complicarse con la muerte de familiares y amigos, lo que podría llevar a reducir el bienestar de aquellas personas que pierden sus contactos laborales, redes sociales e identidades (Santos, 2009); además, puede agravarse con la incapacidad para percibir sus competencias, habilidades y los aspectos positivos del entorno que conllevan a la afectación en la calidad de vida y por ende ausencia de bienestar psicológico.

En este sentido, existen hallazgos empíricos que demuestran que el nivel de autoestima y autosatisfacción decrece con la edad, según lo señala Thompson (2008). Asimismo, es concebido el envejecimiento como un fenómeno de mayor 
impacto en las sociedades modernas (Alvarado y Salazar, 2014). En primer lugar porque, con los avances científicos, aumentan cada vez más las expectativas y posibilidades de vida de los adultos mayores; en segundo lugar, porque esto dificulta en términos económicos la atención de esta población; y en tercer lugar, porque en los países subdesarrollados sigue siendo muy bajo el nivel de aseguramiento social de los adultos mayores; en tal virtud, la Organización de las Naciones Unidas (ONU) en su informe de la Segunda Asamblea sobre el Envejecimiento (2012), propuso un Plan de Acción Internacional sobre el Envejecimiento, basado en direcciones prioritarias: las personas de edad y el desarrollo; la promoción de la salud, el bienestar en la vejez y el logro de entornos emancipadores y propicios.

Así mismo, en el contexto de América Latina, el cambio demográfico continúa invirtiendo la pirámide poblacional en donde las personas adultas mayores aumentan y la tasa de natalidad disminuye, esto sumado a la pobreza y exclusión, se aumentan los riesgos de esta población. Cerca de la mitad o más de la población adulta mayor de América Latina no accede a una jubilación o pensión que le permita afrontar los riesgos y consecuencias generadas por la pérdida de ingresos después de la jubilación, teniendo en cuenta también, que de acuerdo al esquema contributivo de los Sistemas de Seguridad en los países latinoamericanos, las posibilidades de acceder a este tipo de protección social se reduce cada vez más para aquellos grupos poblacionales que se encuentran por fuera del sistema, lo que aumenta la vulnerabilidad. Así, en Colombia, en el censo del DANE del año 2005, se calculó que, de una población total de 41.468 .384 de habitantes, el $6.31 \%$ de la población colombiana corresponde a mayores de 65 años.

Según Romero (2010) sólo 4 de cada 10 personas mayores de 70 años en Latinoamérica, cuentan con ingresos provenientes de alguna jubilación o pensión. Lo cierto es que todas estas transformaciones económicas, sociales, culturales y políticas, hacen cada vez más difícil y problemático el bienestar de las personas adultas mayores que ingresan al proceso de jubilación.

Según Aranda, Pando y Aldrete (2011) este grupo etario al perder la dinámica laboral trata de reestructurar el sistema de roles para insertarse nuevamente en sociedad, en este contexto el tema es relevante psicosocialmente; y desde la salud pública, es necesario prepararse para la jubilación, ya que no todas las personas se adaptan de la misma manera a este proceso. Complementa Aguilera (2010) indicando que múltiples factores intervinientes tales como haber sido forzado a retirarse; problemas de salud, económicos; problemas conyugales, familiares, dependientes enfermos, el locus de control externo, la propia actitud hacia la jubilación y de los apoyos sociales, entre otros pueden convertirse en eventos estresantes que generan sentimientos de depresión, ansiedad, abandono, desesperanza y disgusto con la familia; en este sentido la jubilación si no está asociada al bienestar es un problema de salud pública.

Se señala también la pretensión permanente de medir el bienestar y científicos han creado instrumentos tales como la Escala de Satisfacción con la Vida (Satisfaction With Life Scale o SWLS) (Diener, 1985); así también, el Índice de Bienestar Personal elaborado por Cummins, para medir la satisfacción en diferentes esferas de la vida, incluyendo el nivel de vida, las relaciones humanas y la espiritualidad (International Wellbeing Group, 2006). En este sentido, también se contempla a las Escalas de Bienestar Psicológico de Ryff (1989) (Scales of psychological wellbeing-reduced) cuyas dimensiones considera las características multidimensionales del bienestar (Easterlin, 1974). 
En el Ecuador la población económicamente activa, refleja una tasa del 51.5\% corresponde a empleados y trabajadores; los subempleados son el $43.6 \%$; y desempleados el 4.9\%. La población ecuatoriana que tiene más de 60 años es el 9.3\% este dato estadístico incluye a los profesores universitarios (INEC, 2013).

Al hacer referencia a los docentes universitarios activos con nombramiento de la universidad pública del Ecuador, quienes son un total de 2268 con prevalencia del sexo masculino en una relación de 2:1; más de 62 años tiene el 42,2\%; de 38 a 61 años son el $49.5 \%$; y el 8,3\% menores de 37 años (Cifras UCE, 2018). La planta docente, inició un proceso de renovación generacional, al aplicar el Reglamento de Carrera y Escalafón del Profesor e Investigador del Sistema de Educación Superior, que obligó a la jubilación a los profesores que tienen 70 años o más. En el 2014, se jubilaron más de 200 docentes simultáneamente, cabe resaltar que muchos de ellos, solicitaron jubilación anticipada por no perder otros beneficios sociales; sin embargo, sea cual fuere la causa de la jubilación, este hecho permite avizorar la magnitud del problema de la presente investigación; asimismo, es importante conocer cuál es el grado de bienestar psicológico de esta población vulnerable y cómo se ve afectada la calidad de vida.

Para Keyes, Shmotkin y Ryff (2002) la jubilación es una experiencia positiva, oportuna para disfrutar el tiempo libre y liberarse de los compromisos laborales, es el denominado bienestar psicológico, incluyendo tanto juicios cognitivos como reacciones afectivas. En este sentido Diener et al (1985) el bienestar psicológico es abordado desde una perspectiva fundamentalmente emocional, es la felicidad y satisfacción con la vida. Contrariamente, Arango y Ruiz (2012) manifiestan que la jubilación trae consigo una connotación negativa de creer que cuando se llega a esta etapa, se pierde el rol social asignado y muchas veces se estigmatiza.

En este sentido, la posibilidad de una mejor adaptación a la jubilación está supeditada a factores como la buena salud, ingresos económicos suficientes, la actitud hacia la jubilación y de los apoyos sociales (Aguilera, 2010). En otros casos la jubilación también puede convertirse en unos eventos estresantes por ejemplo el hecho de haber sido forzado a retirarse, la no planificación de la jubilación asociada a los problemas de salud, económicos, conyugales, familiares, entre otros.

Desde el punto de vista de la transición entre el trabajo, la prejubilación y la jubilación, periodo en el cual la persona se enfrenta a cambios propios de la edad y al prejuicio social asociado al envejecimiento, las personas deben recurrir a diversos factores internos y externos para lograr una mejor adaptación, como lo menciona Beck (1984).

El bienestar psicológico, asentido por Foresight Commission británica (2008), es un estado dinámico en el cual el individuo puede desarrollar su potencial, trabajar productiva y creativamente, construir relaciones sólidas y positivas con otros, y contribuir a su comunidad. El bienestar aumenta cuando un individuo puede cumplir sus metas personales, sociales y alcanzar un propósito en la sociedad.

A su vez, Sempere et al (2010) conciben al bienestar psicológico, a la felicidad y al buen vivir, como un objetivo común de todas las sociedades; y refieren que concomitantemente con las necesidades fisiológicas, están las necesidades psicosociales, tales como el reconocimiento, autoestima, seguridad, pertenencia a una comunidad, confianza, mismas que coexisten enmarcados en las normas sociales.

Análogamente, Gubriun (1973) considera que el aspecto social es un factor determinante en el bienestar de los jubilados y enfatiza en los apoyos sociales (entorno), pero también en factores personales, ya que el apoyo social se refiere a los 
aspectos psicosociales y a la ayuda tangible que provee la red social y que recibe la persona. El autor planteó la Teoría del Medio Social o Socioambiental y a partir de estas proposiciones, se han planteado a lo largo del tiempo, varios Modelos Sociológicos de envejecimiento, que intentan explicar el comportamiento de los adultos mayores en proceso de jubilación. En suma, un bajo nivel de apoyo social se asocia a un aumento de la morbilidad y mortalidad especialmente en una población vulnerable.

El estudio tuvo como objetivo analizar el bienestar psicológico asociado a la jubilación en docentes universitarios jubilados de la Universidad Central del Ecuador; para lo cual caracterizó el perfil socio-demográfico de los docentes universitarios jubilados de esta institución de educación superior, entre el 2012 y 2017; se determinó el grado de bienestar psicológico de la muestra de docentes universitarios jubilados de la universidad pública del Ecuador; evaluó el índice de apoyo social; para finalmente asociar el grado de bienestar psicológico con el apoyo social de esta población.

\section{METODOLOGÍA}

Diseño: correlacional, no-experimental, transversal, la aplicación se realizó en un sólo momento de la investigación. No se manipularon variables. Tipo de estudio: cuantitativo explicativo con variables observables, relación funcional entre un predictor y criterio. Se aplicó el método científico, considerando el procedimiento lógico, para obtener un conocimiento válido desde el punto de vista científico. Para generalizar los resultados, se aplicó el método deductivo. Se aplicó las técnicas de la entrevista, estableciendo una relación de comunicación directa cara a cara con el investigador, para orientar el proceso de obtención de la información expresada en respuestas verbales y metalingüísticas del participante; y la técnica psicométrica, para el análisis de los cuestionarios y procesamiento de los resultados.

Los cuestionarios se validaron en una población de 222 adultos mayores del Programa Ciudad Activa Deportiva del Distrito Metropolitano de Quito; se evaluó las versiones en español, para lo cual se conformó un Comité de dos expertos psicolingüistas y un psicólogo, quienes valoraron la equivalencia transcultural, semántica, experiencial e idiomática de los cuestionarios; se calculó el índice de consistencia interna y de validez convergente; así en primer término se validó la Escala de Bienestar Psicológico de Ryff (1995) de 39 ítems con un Alfa global de Cronbach de 0.8444; y sus dimensiones: autoaceptación (0.4296), es el criterio más utilizado para definir bienestar y es conceptualizado como la figura central de la salud mental, como una característica de madurez, realización personal y funcionamiento óptimo; relaciones positivas con los demás (0.2785) la importancia de la calidez y confianza en las relaciones interpersonales, así como la capacidad de amar, son vistas como uno de los principales componentes de la salud mental, siendo también un criterio de madurez; autonomía (0.5336), es la autodeterminación, la independencia y la regulación de la conducta; dominio del entorno (0.4865) es la habilidad de elegir o crear ambientes acordes a las propias condiciones físicas, implica la capacidad de manipular y controlar los entornos o ambientes complejos contando con la participación del medio; propósito en la vida (0.6278) destaca la comprensión de un propósito o significado de la vida, un sentido de dirección o intencionalidad; y crecimiento personal (0.7092) es el funcionamiento psicológico óptimo requiere de un desarrollo de su propio potencial, crecer y expandirse como persona; estado de ánimo equilibrado, cualidades como autenticidad, felicidad, 
alegría, sentimiento de dignidad, esperanza, anhelos y proyectos de vida, en otras palabras, sentirse bien y el buen uso del tiempo libre.

En segundo término, se validó el cuestionario MOS de Apoyo Social Percibido (Sherbourne y Stewart, 1991) consta de 20 ítems, alcanzó un Alfa global de Cronbach 0.908 y las dimensiones apoyo emocional (0.802); ayuda material (0.724); relaciones sociales de ocio y distracción (0.780) y apoyo afectivo (0.689). La dimensión de apoyo emocional y apoyo efectivo se integraron en una sola, porque en el contexto ecuatoriano es similar su constructo. Los parámetros del MOS mostraron valores aceptables, válido para medir la multidimensionalidad.

Población y muestra, docentes jubilados de la universidad pública del Ecuador, durante 6 años consecutivos. Muestra de tipo probabilística; para el cálculo del tamaño de la muestra se aplicó la fórmula para poblaciones finitas, con una $\mathrm{N}=725$; calculado con un porcentaje de confianza de $95 \%$ y un $5 \%$ de error se obtuvo una $\mathrm{n}=255$, más un $10 \%$ de no respuesta la muestra alcanzó el tamaño de 280 docentes jubilados; mismos que cumplieron los criterios de inclusión (Docentes jubilados en el período 2012 al 2017 con contratos indefinidos de cualquiera de las categorías académicas y tiempo de dedicación y que voluntariamente quieran participar en la investigación). Se excluyeron docentes jubilados que padezcan algún trastorno o enfermedad que incapacite responder los cuestionarios.

La presente investigación involucró a seres humanos, por tanto, se consideraron aspectos éticos y legales, que salvaguarden la integridad física, psicológica y social de los sujetos participantes en el proceso de la investigación.

Procedimiento: Previa la autorización y registro del protocolo de la investigación en las instancias pertinentes se obtuvo la base datos directamente de los archivos de la Unidad de Gestión de Personal.

Se validaron los dos cuestionarios en una población de similares características como se detalló anteriormente. Se realizó el cálculo del tamaño de la muestra con la fórmula para poblaciones finitas. Se contactó vía mail a los docentes jubilados; además, se estableció una cita; se realizó la inducción, entrevistas y aplicación de los cuestionarios. A otra parte de la población se la contactó en la Universidad del Adulto Mayor de la universidad pública del Ecuador. Finalmente, se realizó la tabulación, análisis de resultados, discusión conclusiones.

\section{RESULTADOS}

Para la estadística descriptiva se consideró el total de docentes jubilados registrados en la base datos de docentes jubilados de la universidad pública del Ecuador, en el período de 2012-2017 que fueron 725 personas, con un promedio de edad de 67 años. Del total de docentes el número de hombres son mucho mayor que el número de mujeres; en una relación de 9:1, según Tabla 1.

Tabla 1: Docentes jubilados 2012-2017 según género en la universidad pública del Ecuador

\begin{tabular}{crr}
\hline Género & Frecuencia & Porcentaje \\
\hline Hombres & 663 & 91 \\
Mujeres & 62 & 9 \\
Total & $\mathbf{7 2 5}$ & $\mathbf{1 0 0}$
\end{tabular}

Fuente: Unidad de Gestión de Personal Docente, Administrativo y de Servicios de la universidad pública del Ecuador. 
Según el año de jubilación, la frecuencia y porcentaje reflejan que el año 2014 y 2013 , fueron los que mayor porcentaje de docentes jubilados en la Universidad pública del Ecuador (Tabla 2).

Tabla 2: Docentes jubilados por año calendario

\begin{tabular}{rrr}
\hline Meses & Frecuencia & $\%$ \\
\hline $\mathbf{2 0 1 2}$ & 194 & 19 \\
$\mathbf{2 0 1 3}$ & 297 & 27 \\
$\mathbf{2 0 1 4}$ & 23 & 41 \\
$\mathbf{2 0 1 5}$ & 25 & 3.18 \\
$\mathbf{2 0 1 6}$ & 48 & 3.82 \\
$\mathbf{2 0 1 7}$ & $\mathbf{7 2 5}$ & 6 \\
Total & & $\mathbf{1 0 0}$
\end{tabular}

Fuente: Unidad de Gestión de Personal Docente, Administrativo y de Servicios de la universidad pública del Ecuador.

Según el tipo de jubilación, la mayoría de los docentes se retiraron voluntariamente y por obligación; tal como se aprecia en la tabla 3.

Tabla 3: Tipo de jubilación

\begin{tabular}{lrr}
\hline \multicolumn{1}{c}{ Tipo } & Frecuencia & $\%$ \\
\hline Jubilación por invalidez & 1 & 0,1 \\
Reffinitick. & 138 & 19,0 \\
Renuncia & 256 & 35,3 \\
Retiro obligatorio & 289 & 39,9 \\
Retiro voluntario & 41 & 5,7 \\
a la iubilación. para acogerse & 725 & 100
\end{tabular}

Fuente: Unidad de Gestión de Personal Docente, Administrativo y de Servicios de la universidad pública del Ecuador

La aplicación de los cuestionarios se realizó a la muestra probabilística de 280 docentes y se obtuvieron los siguientes resultados:

De la muestra de estudio en relación con el estado civil 7 de cada 10 docentes jubilados se encuentran casados; 2 de cada 10 han enviudado, y asimismo 1 de cada 10 viven en unión libre o son solteros, tal como se aprecia en el gráfico 1

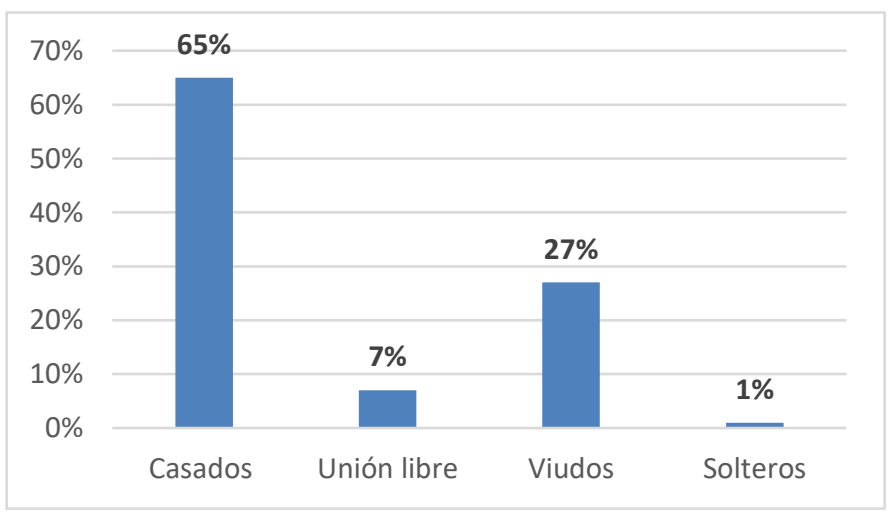

Gráfico 1: Distribución de docentes jubilados según estado civil 
Consecuentemente, según su condición mayoritariamente viven con su pareja o familiares cercanos según se aprecia en el gráfico 2

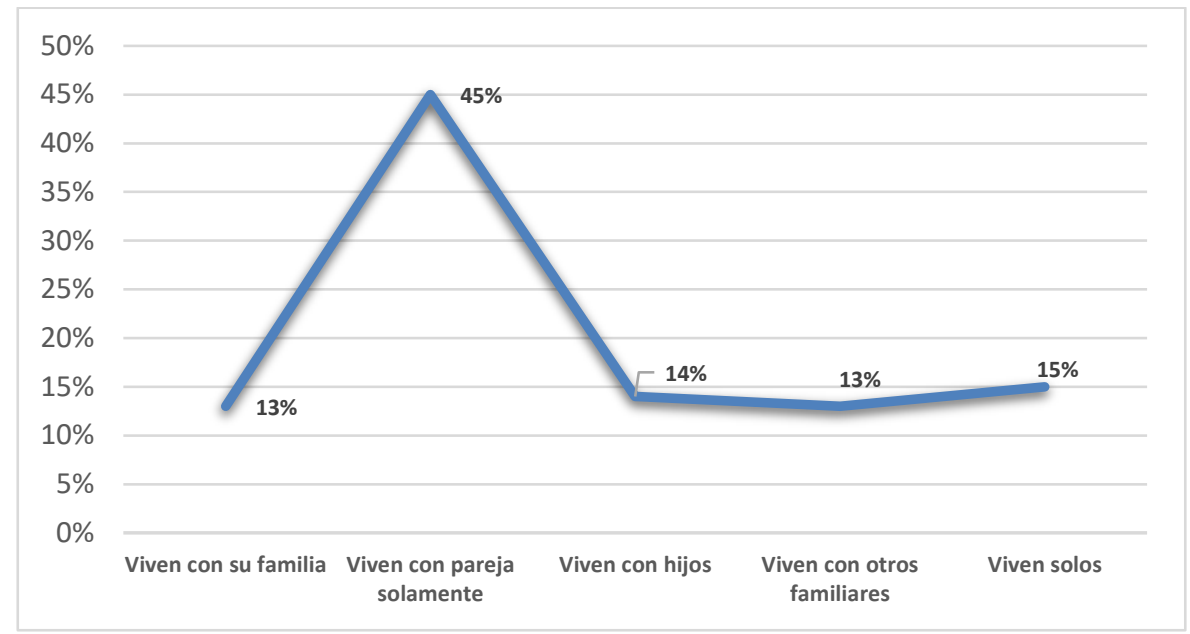

Gráfico 2: Distribución de la población según condición

Del análisis del apoyo social (social support) en la muestra de estudio, se evidenció un elevado índice de apoyo social (80/94) puntos; es decir, que cuentan con la ayuda tangible o asistencia que se da a través de las transacciones interpersonales. La población en referencia se encuentra bien informada, cuentan con personas que les aconsejen y les den sugerencias para resolver los problemas $u$ otras adversidades vitales estresantes. Además, en la parte emocional, tienen personas empáticas cercanas que les expresan sentimientos de amor, confianza y preocupación, especialmente desde su núcleo familiar; aunque también en un grupo minoritario, demuestra fuentes de apoyo externo, como son los amigos, la comunidad y otros actores (Tabla 4).

Tabla 4: Índice de Apoyo Social (MOS)

\begin{tabular}{lccc}
\multicolumn{1}{c}{ Componentes } & \multicolumn{2}{c}{ Puntaje baremizado } \\
\hline Índice Global de Apoyo Social & $\mathbf{8 0}$ & $\mathbf{2 0}$ & $\mathbf{9 4}$ \\
Apoyo Emocional/afectivo & 49 & 8 & 55 \\
Ayuda Material & 13 & 4 & 20 \\
Relaciones Sociales de Ocio y Distracción & 18 & 4 & 20
\end{tabular}

De la evaluación del grado de bienestar psicológico global moderado alcanzaron un puntaje de 142 puntos lo que corresponde a un bienestar psicológico elevado según la escala Ryff(1995).

Siendo la dimensión relaciones positivas con otros, la más alta lo que implica fuerte empatía, relaciones estrechas y cálidas con los demás, demostración de afecto. Seguida de dominio del entorno; es decir, control de actividades, capacidad de elegir contextos y sensación de control y competencia; a diferencia de propósito en la vida, que es la baja, lo que indica sensación de estar sin rumbo en la vida (Gráfico 3). 


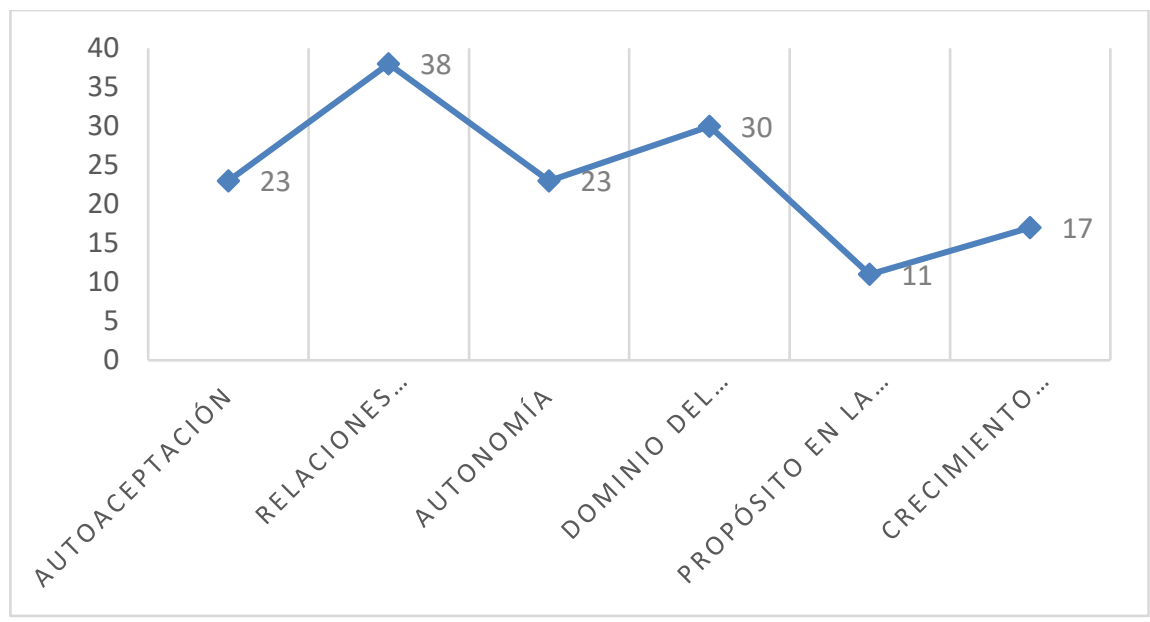

Gráfico 3: Dimensiones del bienestar psicológico (Riff)

Del análisis de la tabla cruzada de doble entrada con las dimensiones de los dos cuestionarios aplicados, se deduce que: la mayor parte de los docentes universitarios jubilados se manifiestan en las Relaciones Positivas y Dominio del Entorno, expresadas por el Apoyo Emocional/Afectivo; las demás dimensiones cruzadas tienen similares frecuencias; a diferencia del Propósito en la Vida y Autonomía con Ayuda Material (Tabla 5).

Tabla 5: Bienestar Psicológico * Apoyo Social

\section{Apoyo Social (MOS)}

Apoyo Ayuda Material Relaciones Total

Emocional/afectivo Sociales de Ocio

\begin{tabular}{|c|c|c|c|c|c|c|c|c|c|}
\hline & \multirow[b]{2}{*}{ DIMENSIONES } & \multirow[b]{2}{*}{ Frec. } & \multirow[b]{2}{*}{$\%$} & \multirow[b]{2}{*}{ Frec. } & \multirow[b]{2}{*}{$\%$} & \multicolumn{2}{|c|}{ y Distracción } & \multirow[b]{2}{*}{ Frec. } & \multirow[b]{2}{*}{$\%$} \\
\hline & & & & & & Frec. & $\%$ & & \\
\hline \multirow{7}{*}{$\begin{array}{c}\text { Bienesta } \\
r \\
\text { Psicológ } \\
\text { ico } \\
\text { (RYFF) }\end{array}$} & Autoaceptación & 17 & 11 & 6 & 11 & 13 & 18 & 36 & \\
\hline & $\begin{array}{l}\text { Relaciones } \\
\text { positivas }\end{array}$ & 47 & 31 & 14 & 26 & 19 & 26 & 80 & \\
\hline & Autonomía & 22 & 14 & 5 & 9 & 4 & 5 & 31 & \\
\hline & $\begin{array}{l}\text { Dominio del } \\
\text { entorno }\end{array}$ & 29 & 19 & 13 & 25 & 15 & 21 & 57 & \\
\hline & $\begin{array}{l}\text { Propósito en la } \\
\text { vida }\end{array}$ & 18 & 12 & 6 & 11 & 8 & 11 & 32 & \\
\hline & $\begin{array}{l}\text { Crecimiento } \\
\text { personal }\end{array}$ & 21 & 14 & 9 & 17 & 14 & 19 & 44 & \\
\hline & Total & 154 & 100 & 53 & 100 & 73 & 100 & 280 & \\
\hline
\end{tabular}

Mediante el análisis estadístico inferencial de las correlaciones, se debela alta correlación entre el índice global del apoyo social (MOS) y sus componentes. Mientras que en la Escala de Bienestar Psicológico (RYFF) la Autoaceptación tiende a estar fuertemente correlacionado con los otros componentes excepto con relaciones positivas y autonomía (Tabla 6). 
Tabla 6

Matriz de correlaciones

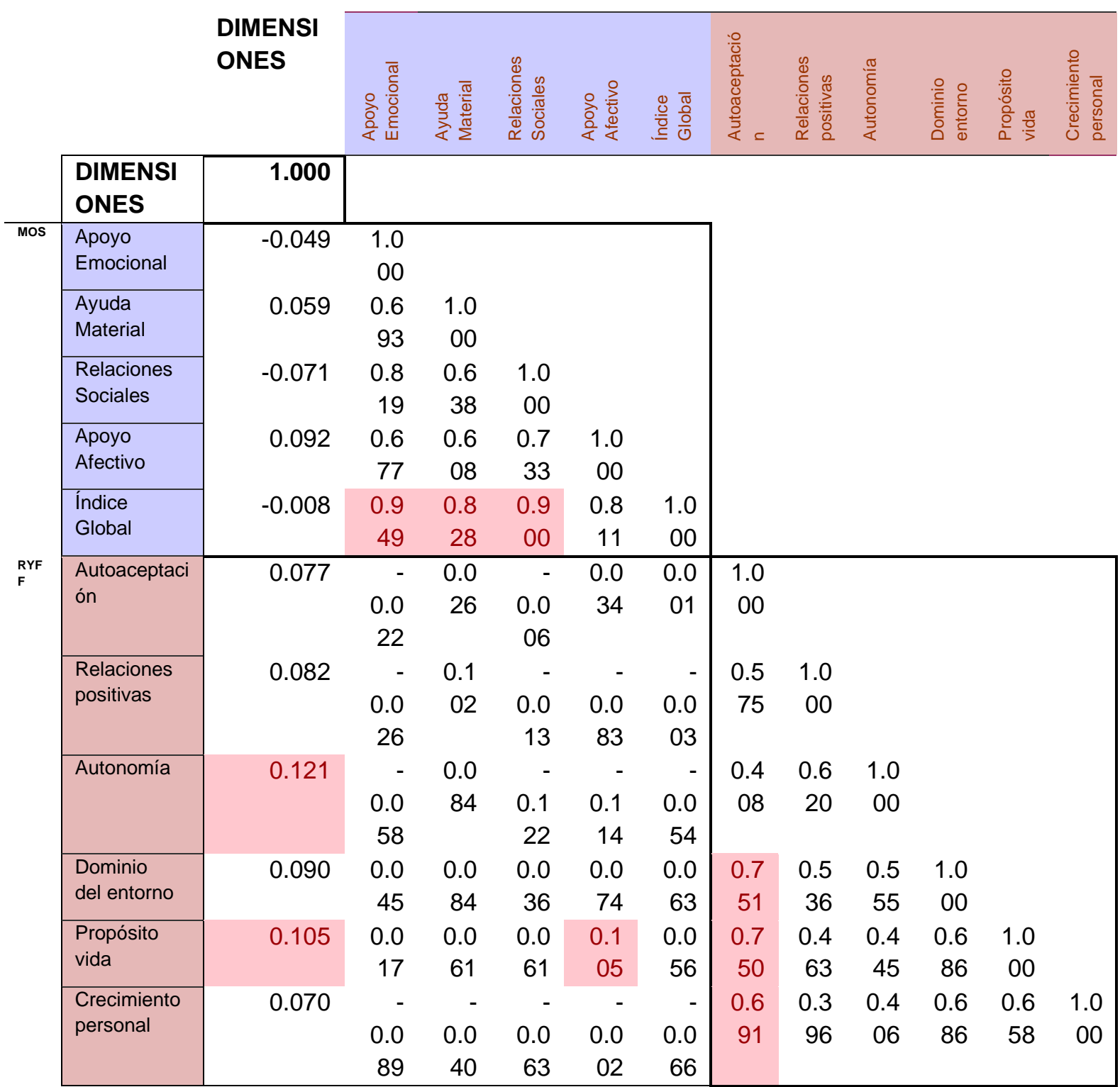

\section{DISCUSIÓN}

A la luz de los resultados se discute los hallazgos del presente estudio; así la OIT, refiere que alrededor de 600 millones de personas (10\%, aproximadamente) en el mundo son adultos mayores (Martínez, 2009) y en su Informe Anual 2014, indicó que el $52 \%$ de los jubilados del mundo no reciben una pensión adecuada, por tal motivo muchos adultos mayores optan por seguir trabajando, con una remuneración baja, por lo que el bienestar psicológico se ve afectado; datos contrarios a los reflejados en la población de este estudio, donde su remuneración está garantizada, lo cual constituye un componente del bienestar psicológico.

La Universidad del Valle ha realizado varios estudios sobre esta temática, así por ejemplo: "Caracterización y nivel de satisfacción de los jubilados de una institución universitaria del suroccidente colombiano en sus actividades de la vida diaria durante los últimos cinco años" en el año 2013, "Caracterización desde el movimiento, la comunicación y ocupacional de los Jubilados activos de la Universidad le Valle que 
asisten al Servicio Médico Institucional" en el año 2015- 2106. "Caracterización de los roles ocupacionales asumidos por las jubiladas de la universidad del valle en los últimos cinco años", Diseño de un programa de voluntario para la población jubilada activa de la universidad del valle "En pro de nuestro mañana". Lo que demuestra la importancia de esta temática en la región. Específicamente en la Universidad del Valle, Institución de Educación Superior de Carácter Público, existe el programa de Programa de Atención Integral al Jubilado, el cual depende de la Sección de Desarrollo Humano y Promoción Socioeconómica de la Vicerrectoría de Bienestar Universitario. El programa está dirigido a los pensionados de la Universidad del Valle y a personas mayores de 50 años beneficiarias del Servicio del Salud.

En Chile, la jubilación es legalmente voluntaria y de acuerdo con la Ley 10.383, se considera un derecho de los trabajadores el poder percibir una pensión de vejez para los hombres al cumplir los 65 años y para las mujeres a los 60 años; a diferencia de la ley ecuatoriana que es igual para hombres y mujeres.

En Chile el Instituto Nacional de Estadística (INE, 2016), determinó el $76.1 \%$ trabajadores que se jubilan son hombres y $34.1 \%$ son mujeres, relación similar a los datos de la presente investigación donde también el número de docentes que se jubilan son hombres.

Estudios plantean la pérdida de roles y estatus asociada a la jubilación y sus efectos en la salud psicológica de las personas y enfatiza la importancia de abordar aspectos psicológicos entre las consecuencias de la jubilación, tales como el bienestar y la satisfacción con la vida (Bradbum, 1969); mismas motivaciones que generaron este estudio.

Una de las teorías que aborda el proceso de la jubilación es la de Atchley (1982) quien basó sus postulados en la continuidad y establece que se debe preparar para la jubilación; así mismo se debe familiarizarse con los aspectos implícitos como las actitudes de las personas, la familia e ingresos económicos, entre otros; esta teoría señala siete fases de este proceso: prejubilación, luna de miel, descanso - relajación, desencantamiento, reorientación, rutina y terminación; postulado coincidente con la conclusión del presente estudio, que refiere que hay que prepararse para aceptar y adaptarse a la jubilación para tener calidad de vida en esta etapa vulnerable del ciclo vital.

Desde otra perspectiva Kahneman (1999) propuso al bienestar en niveles, a saber: externo (objetivo-bienes materiales); subjetivo (satisfacción e insatisfacción); del humor persistente (optimista/pesimista); inmediatos (placeres/dolores); estados emocionales transitorios (goce y rabia); y los bioquímicos (bases neuronales del comportamiento); dimensiones diferentes a las abordadas en este tratado.

Por su parte, Gottlieb (1983) concibe al apoyo social como: "información verbal y no verbal, ayuda tangible o accesible dada por los otros o inferida por su presencia y que tiene efectos conductuales y emocionales beneficiosos en el receptor" ( $p$ 209) concepciones similares a las conclusiones del presente estudio.

Estudios realizados en pacientes en hemodiálisis crónica (De los Ríos et al 2005) muestran que un alto grado de apoyo social percibido implica esperanza, percepción de competencia física y psíquica, capacidad de encontrarle el lado positivo a situaciones difíciles, capacidad de relacionarse con otros y de expresar sus frustraciones; respecto a la familia, el $92.4 \%$ de los pacientes refirió contar siempre con su ayuda para todo tipo de problemas (emocionales, económicos, personales y cotidianos). De los pacientes que relataron tener amigos, el 55.7\% sentía que contaba siempre con su apoyo, principalmente en aspectos emocionales; resultados 
coincidentes con la percepción de apoyo social desde su red social en docentes universitarios jubilados.

\section{CONCLUSIONES}

Se caracterizó el perfil sociodemográfico de los docentes jubilados de la universidad pública del Ecuador en el período de 2012-2017 y fueron 725 personas. El promedio de edad es de 67 años. El 68\% de docentes, se jubilaron entre el 20132014. El $75 \%$ fue por retiro voluntario y obligatorio. En su mayoría (7:10) están casados; han enviudado (2:10), y en unión libre o son solteros (1:10). Según su condición, aproximadamente la mitad de la población (45\%) estudiada vive solamente con su pareja y el resto con otros familiares.

Se determinó en los docentes jubilados de la universidad pública del Ecuador, un grado de bienestar psicológico elevado; destacándose las Relaciones Positivas con Otros, el Dominio del Entorno; sensación de control y competencia; a diferencia de Propósito en la vida que obtuvieron un bajo grado. En relación con la otra variable se demostró un elevado índice de apoyo social (80/94) puntos; la muestra estudiada con personas empáticas cercanas que les ayudan y dan apoyo emocional frente a problemas.

Finalmente, la jubilación representa un hito en el ciclo vital que plantea diversos desafíos, por tanto, el estudio recomienda diseñar un programa de acompañamiento para mejorar la adaptación al proceso de jubilación de los docentes universitarios para incrementar el nivel de bienestar psicológico y el apoyo social afectivo, en pro de fomentar la salud mental de esta población vulnerable.

\section{AGRADECIMIENTOS}

Universidad del Adulto Mayor de la universidad pública del Ecuador; Programa Ciudad Activa Deportiva del Distrito Metropolitano de Quito.

\section{BIBLIOGRAFÍA}

Alvarado, A. y Salazar, A. (2014). Análisis del concepto de envejecimiento. Revista Gerokomos vol.25 no.2 Barcelona, España.

Aguilera, M.A. (2010). Salud y Jubilación. Jalisco, México: Editorial Página seis S.A.

Aranda, B., Pando, M. y Aldrete, R. (2001). Jubilación, depresión y redes de apoyo social al adulto mayor en la Zona Metropolitana de Guadalajara. En Revista Investigación y Salud. 3(3); Diciembre, pp. 193-1999.

Arango, V. y Ruiz, I. (2012). Diagnóstico de los Adultos Mayores. Fundación Saldarriaga Concha. www.saldarriagaconcha.org recuperado el 15 de febrero de 2012.

Atchley, R.C. (1982). Retirement: Leasing the world of work. The Annals of the American Academy of Policy and Social Science, 464, 120-131.

Beck, S.H. (1984). Retirement preparation programs: Differentials in opportunity and use. Journal of Gerontology, 39, 596-602. 
De los Ríos JL., Sánchez JJ., Barrios P., Avila TL. Quality of life in patients with diabetic nephropathy. Invest educ enferm 2005; 23(1): 34-41 recuperado de:

http://www.scielo.org.co/pdf/iei/iee/v23n2/v23n2a03.pdf

Diener, E., Emmons. RA., Larsen, RJ y Griffin, S. (1985). 'The Satisfaction With Life Scale' Journal of Personality Assessment 49:71-75.

Easterlin, R. (1974). Does economic growth improve the human lot? Nations and households in economic growth: Essays in honor of Moses Abramowitz. New York: In P David \& M Reder (eds) Academic Press.

Foresight Mental Capital and Well-being Project (2008). Foresight Mental Capital and Well-being Project: Final Project Report. London: The Government Office for Science.

Gottlieb, B.H. (1983). Social support strategies: Guidelines for mental health practice. Beverly Hills: Sage Publications.

Kahneman, O. (1999). Objective happiness. In Well-being: the foundations of hedonic psychology New York: ed. E.

Keyes, C. Shmotkin y Ryff. (2002). Optimizing Well-Being: The Empirical Encounter of Two Traditions. Journal of Personality and Social Psychology (82)6, 1007-1022.

Organización Mundial de la Salud (2012). Informe de la Segunda Asamblea Mundial sobre el Envejecimiento. Madrid, 8 a 12 de abril de 2002. Recuperado de: http://www.un.org/es/comun/docs/?symbol=A/CONF.197/9. pp. 85

Pando, M. Ruiz, D. Parra y Aranda, C. (2013). Eugenesia Laboral. Salud Positiva en el Trabajo. Cali, Colombia: Universidad Libre Seccional.

Ryff, C. D. (1989). Happiness is everything, or is it? Explorations on the measuring of research on hedonic and eudaimonic well-being. Annual Review of Psychology (52) 141-166. DOI: http://dx.doi.org/10.1037//0022-3514.57.6.1069

Romero, X. (2010). La reducción de la pobreza en la vejez, una tarea pendiente para el logro de los Objetivos de Desarrollo del Milenio en América Latina. Red Latinoamericana de Gerontología. N.124 Recuperado de: https://www.gerontologia.org/portal/information/showlnformation.php?idinfo=1795

Sánchez, P. (2000), Empleo, flexibilidad de la jubilación e ideología. Breve cronología del debate en España. Revista del Ministerio de Trabajo y Asuntos Sociales. España.

Sempere, J., Acosta, A., Abdallah y Ortí, M. (2010). Enfoques sobre bienestar y buen vivir. Madrid: Editorial CIP-Ecosocial.

Thompson, S y Marks N (2008). Measuring well-being in policy: Issues and applications. Disponible en www.neweconomics.org/programmes/well-being 